\title{
A Evolução do Conceito de Operante
}

\author{
João Cláudio Todorov ${ }^{1}$ \\ Universidade Católica de Goiás \\ Universidade de Brasília
}

\begin{abstract}
RESUMO: O conceito de operante surge nos anos 30 como necessidade, dada a dificuldade encontrada por Skinner para analisar seus dados usando como ferramenta o conceito de reflexo. O presente trabalho apresenta a evolução do conceito até o presente, quando a unidade de análise não tem mais a ver com a estrutura do comportamento: a nova ferramenta é o conceito de contingência tríplice.
\end{abstract}

Palavras-chave: resposta; operante; contingência.

\section{The Evolution of the Concept of Operant}

\begin{abstract}
The concept of operant was developed by Skinner as an answer to the problems he had using the concept of reflex to describe the eating behavior of rats. The present work shows the evolution of the concept to present times, when the unity of analysis is the concept of triple contingency.
\end{abstract}

Key words: response; operant; contingency.

Honig e Staddon (1977), na introdução do livro que organizaram, abrem o primeiro parágrafo com uma afirmação que motivou este trabalho; "Mais de dez anos se passaram desde a publicação de "Operant behavior" (Honig, 1966), que foi um primeiro esforço para providenciar um relato relativamente compreensivo daquelas áreas do pensamento e da pesquisa na psicologia que foram influenciadas substancialmente por métodos operantes."

Os autores resumem a contribuição de Honig (1966) e a deles próprios (Honig \& Staddon, 1977) como contribuição à metodologia de pesquisa. É certo que uma grande contribuição de Skinner foi o desenvolvimento de pesquisas com $n=1$, ou seja, sem grupos experimentais ou grupos de controle (Sidman, 1960). É certo também que a contribuição teórica de Skinner $(1938 ; 1953 ; 1966$ : 1974) independe da metodologia de pesquisa que desenvolveu. Sua primeira grande contribuição nada tem a ver $\operatorname{com} n=1$. É puramente conceitual sua definição de reflexo (antes mesmo de propor a distinção entre operantes e respondentes (1935a).

O sucesso dos métodos operantes na psicofarmacologia (Todorov, 1981), na psicologia clínica (Lettner \& Rangé, 1988; Banaco, 1997), entre outras áreas, tende a esconder a importância do desenvolvimento conceitual. As posições encontradas em Honig (1966) e Honig e Staddon (1977) parecem refletir um estágio no desenvolvimento dos conceitos da análise do comportamento, dificuldades que foram paulatinamente vencidas por reformulações e avanços teóricos. O presente trabalho se propõe como uma breve história da evolução do conceito de operante, reconhecendo que o tema operante não é original (ver, por exemplo, Schick, 1971;

1 Endereço: SHIN QI 01 Conj. 09 Casa 11 71505-090 Brasília, DF. email: todorov@unb.br
Catania, 1973; Sério, 1983; 1990), ligando os trabalhos iniciais de Skinner nos anos 30 às contribuições atuais (Catania, 1996; Hayes, Gifford \& Wilson, 1996; Simonassi, 2001).

Em "The behavior of organisms" Skinner (1938) aprimora a distinção entre operantes e respondentes apresentada em trabalhos anteriores (Skinner, 1935b; 1937). É ainda uma definição sofrível: operante é todo comportamento para o qual não se identifica um estímulo eliciador, como nos reflexos de Pavlov. O operante de então é como uma cesta de lixo: o que não couber na definição de respondente é classificado como operante. Mas é um primeiro passo:

O tipo de comportamento correlacionado com estímulos eliciadores especificos pode ser denominado de comportamento respondente e uma correlação determinada um respondente. Pretende-se com isso que o termo tenha o sentido de uma relação com um evento prévio. Refiro-me ao comportamento que não está sob esse tipo de controle como operante e a qualquer exemplo especifico como um operante. (Skinner, 1938, p. 20).

Voltando a Honig e Staddon (1977), depois de duas páginas comentando os prós e contras da metodologia operante, citando Sidman (1960) inclusive, os autores afirmam: "O operante ainda é uma unidade verdadeiramente viável, ... , mas não pode ser separado de outras formas de comportamento". Meia dúzia de linhas depois, Honig e Staddon já falam de outra coisa: "comportamento operante é estudado providenciando-se que o animal afete seu ambiente de alguma maneira ... Esse efeito, que pode ser conseguido de qualquer maneira que o animal escolha, é chamado de resposta.". Se resposta é um exemplo de comportamento operante, então o que é um operante? Pela definição acima, comportamento operante é um artifício experimental, observado em animais no laboratório. Mas o que é um operante, uma "unidade verdadeiramente viável"? 
Antes de voltar a essa questão, é interessante verificar o caminho percorrido por Skinner de 1938 a 1974. Em seu primeiro livro (Skinner, 1938), como vimos, operante é tudo o que não for respondente. Em 1953, em "Science and human behavior”, o conceito já está mais elaborado:

Uma resposta que já ocorreu não pode, é claro, ser prevista ou controlada. Apenas podemos prever a ocorrência futura de respostas semelhantes. Desta forma, a unidade de uma ciência preditiva não é uma resposta, mas sim uma classe de respostas. Para descrever-se esta classe usar-se-á a palavra "operante". O termo dá ênfase ao fato de que o comportamento opera sobre o ambiente para gerar consequências. As consequências definem as propriedades que servem de base para a definição de semelhança de respostas. O termo será usado tanto como adjetivo (comportamento operante) quanto como substantivo para designar o comportamento definido para uma determinada conseqüência. (Skinner, 1953/1967, p. 71).

Em outras palavras, praticamente a mesma definição de operante é dada em "Verbal behavior" (Skinner, 1957):

Os tipos de comportamento nos quais estamos geralmente interessados têm, como temos visto, um efeito sobre o ambiente o qual tem um efeito de retorno sobre o organismo. Tal comportamento se distingue de atividades principalmente concernentes à economia interna do organismo quando denominamos de "comportamento operante" as atividades que operam sobre o ambiente. Por conveniencia, qualquer unidade de tal comportamento denomina-se "um operante". Na maioria dos casos "operante" é intercambiável com o termo tradicional "resposta", mas os termos nos permitem fazer uma distinção entre uma instância de comportamento ("Fulano fumou um cigarro entre 14:00 e 14:10 ontem") e um tipo de comportamento (fumar cigarros). O termo "resposta" freqüentemente é usado em ambos os casos ainda que não se aplique facilmente ao segundo significado. A descrição de uma instância do comportamento não requer a descrição de variáveis a ela relacionadas ou de uma relação funcional. O termo operante, por outro lado, está relacionado à previsão e ao controle de um tipo de comportamento. Ainda que observemos apenas instâncias, estamos interessados nas leis que especificam os tipos. (Skinner, 1957, p. 20)

"Ciência e comportamento humano" foi escrito durante um intervalo da peleja que foi escrever "Comportamento verbal”: Skinner começou o trabalho em 1934, parou durante a Segunda Guerra Mundial (1941-1944), e parou de novo para escrever "Ciência e comportamento humano" no fim dos anos 40. O trecho acima, contudo parece ter sido escrito depois. Em "Comportamento verbal" está muito clara a preocupação de Skinner com evitar o formalismo. Aos invés da forma, ou da estrutura do comportamento, no operante o que interessa é a relação. Esse caminho de Skinner, dos anos 30 aos anos 50, foi muito bem percorrido por Sério $(1983,1990)$ em dois trabalhos de fôlego.

Nos anos 60 a proposta de Skinner, de dividir o comportamento dos organismos em apenas dois tipos, começa a ser questionada mesmo por pesquisadores que se identificam com seu trabalho. Verhave (1966) organizou uma coletânea de textos de vários autores para ser utilizada como apoio a cursos que usassem "Princípios de psicologia" (Keller \& Schoenfeld, 1950). No primeiro capítulo, "Uma introdução à análise experimental do comportamento" Verhave recorre até a Aristóteles para deixar claro que não vê uma dicotomia respondente/operante, mas sim um continuum:

Se a discussão acima dos comportamentos respondente e operante enfatizou suas diferenças, qualquer implicação de que uma dicotomia absoluta e rígida existe deve ser corrigida. Muitas formas de comportamento ... em certos aspectos ocupam uma posição intermediária entre o reflexo clássico e o operante livre típico ..." ... "Parafraseando Aristóteles, a gradação do reflexo ao operante é contínua. (Verhave, 1966, p. 19-20).

Teitelbaum ilustra um dos usos do conceito de operante que aproveita a metodologia da análise do comportamento, mas com uma definição que se afasta da abordagem de Skinner:

As características do operante (a natureza do ato e do estímulo que o elicia, arbitrária e essencialmente intercambiável, assim como a medida do controle que o animal exerce sobre a resposta) o distinguem como um ato voluntário. ... Isto é tudo que o condicionamento operante tem em comum: a motivação do animal para a obtenção do reforço.... Se um operante ocorre, a motivação existe. (Teitelbaum, 1966, p. 567).

Em 1966 Skinner já parece avançar para uma posição diferente, que por certo foi influenciada por trabalhos como aqueles reunidos por Verhave (1966), mas que poderia ser alcançada apenas pelos seus trabalhos dos anos cinqüenta: além de "Ciência e comportamento humano", no qual a linguagem da análise do comportamento primeiro apresenta os conceitos básicos para depois mostrar como o comportamento humano pode ser visto pelo prisma desses conceitos, e de "Comportamento verbal", um esforço teórico que aprofunda uma parte do livro anterior, Skinner publica o resultado de uma parceria de anos com C. B. Ferster, "Schedules of reinforcement", onde é empiricamente explorada uma impressionante gama de possibilidades para contingências de reforço (Ferster \& Skinner, 1957). A definição de operante dos anos 50 parece insuficiente para os próprios dados e análises daqueles tempos. No capítulo introdutório do livro de Honig (1966) Skinner escreve:

Estamos interessados no comportamento de um organismo por seus efeitos no ambiente. (Um efeito no ambiente social é, obviamente, o despertar de nosso interesse.) (p. 12).

Reflexos eram obviamente "adaptativos", mas este foi primeiramente um efeito filogenético. O termo "operante" foi introduzido para distinguir entre reflexos e respostas que operam diretamente sobre o ambiente (Skinner, 1937) (p. 15).

A solução de Thorndike provavelmente foi sugerida pelo tratamento que Darwin dá ao propósito filogenético. Antes de Darwin se poderia dizer que o propósito de um olho bem desenvolvido era o de permitir ao organismo ver melhor. O princípio da seleção natural moveu o "ver melhor" do futuro para o passado: organismos com olhos bem desenvolvidos descendiam daqueles que foram capazes de ver melhor e por isso produziram mais descendentes. (p. 13). 
Em resumo, no campo do comportamento como um todo, as contingências de reforço que definem o comportamento operante estão por toda parte. Aqueles sensiveis a esse fato à vezes ficam embaraçados com a freqüencia com a qual eles vêem reforço por toda parte, como os marxistas vêem a luta de classes ou os freudianos o complexo de Édipo. (p. 31).

Em "Contingencies of reinforcement" de 1969 Skinner destaca o papel das contingências de reforço na definição de operante:

Um operante é uma classe, da qual uma resposta é uma instância ou membro. ... É sempre uma resposta à qual um reforço é contingente, mas é contingente às propriedades que definem a pertinência a um operante. Assim, um conjunto de contingências define um operante. (Skinner, 1969, p. 131).

A substituição da dicotomia respondente (tendo a ver com a economia interna do organismo) versus operante (agindo sobre o ambiente externo) pela análise que utiliza os conceitos de contingências filogenéticas e contingências ontogenéticas vai acontecendo aos poucos (Skinner, 1953; 1966; 1969). Na citação acima, o que define o operante nada tem a ver com estrutura da resposta ou com a consequência dessa resposta sobre o ambiente. A definição está nas contingências. Em 1974, em "Sobre o behaviorismo", o esforço vai todo para o papel dessas contingências:

Além dos detalhes do comportamento resultante, há boas razões para distinguir entre dois tipos de contingências ... Contingências de reforço levam vantagem em relação a previsão e controle. As condições sob as quais uma pessoa adquire comportamento são relativamente acessíveis e com frequência podem ser manipuladas; as condições sob as quais a espécie adquire comportamento estão quase fora de alcance. Uma conseqüencia desafortunada é que às vezes origens genéticas se tornam um tipo de aterro sanitário; qualquer aspecto do comportamento que no momento não pode ser explicado em termos de contingências de reforço tende a ser atribuido à herança genética.... (Skinner, 1974/1982, p. 44).

Portanto, o comportamento resultante é um detalhe. $\mathrm{O}$ importante é conhecer as contingências que produzem esse comportamento. E voltando à lata de lixo: o que não conseguimos explicar por contingências ontogenéticas corre o risco de ser explicado como resultado da filogênese. As restrições levantadas por Verhave (1966) à dicotomia operante/respondente estão resolvidas. No lugar de um continuum onde respondente e operante são os pontos extremos, exemplos de comportamentos que não se encaixavam na definição são tratados de outra maneira. Skinner (1974/1982) utiliza a sobreposição (ou não) de contingências de reforço e contingências filogenéticas para explicar o que seus críticos apontavam como falhas da análise do comportamento, processos que não podem ser explicados pela dicotomia operante/ respondente: imprinting, imitação (e o instinto de manada), territorialidade e agressão, "universais" específicos da espécie (linguagem, complexo de Édipo). A partir dos anos 70 Skinner não mais se ocupa da definição de operante, mas o assunto é retomado por outros autores.
A unidade de análise: de atos que produzem o mesmo efeito no ambiente ao conceito de unidade funcional.

Schick (1971) examinou as diferentes definições de operante usadas por Skinner desde sua resposta a Konorski \& Miller (1937) até "Contingencies of reinforcement" de 1969 concluindo que todas são insuficientes. Alguns operantes são definidos pela forma da resposta, outros pela característica de produzir reforço (função), outros ainda pela especificação do estímulo discriminativo da contingência tríplice (Skinner, 1937; 1953; 1969; Todorov, 1991). Schick conclui:

Operantes podem ser definidos por propriedades da resposta de diferentes tipos: propriedades de terem certos efeitos, propriedades de ter certas formas, e propriedades de ocorrer na presença de certos estímulos. (Schick, 1971, p. 422).

Assim, enquanto Skinner se afasta da definição de operante e passa a dar ênfase no conceito de contingência, Schick chama a atenção para as características do comportamento que é parte da contingência. É interessante verificar como o conceito de propriedade da resposta é ampliado para incluir a produção do reforço e os estímulos presentes quando a resposta é reforçada.

Catania (1973a, 1973b) retoma a iniciativa de Schick em dois artigos publicados no mesmo ano, um sobre as psicologias da estrutura, da função e do desenvolvimento, outro sobre o conceito de operante. Com Catania, operante discriminado é redundância: todo operante é discriminado. Catania mantém a ênfase de Skinner na contingência tríplice mas volta, como Schick, às propriedades da resposta. Todo comportamento pode ser visto sob os ângulos de sua estrutura (as propriedades formais), de sua função, e do desenvolvimento de estrutura e função. Catania aprofundou essas colocações ao longo do tempo (Catania, 1978, 1988, 1998/ 1999), mas a aceitação de seu ponto de vista não foi geral. Baum, ainda último, mantém uma definição antiga:

Uma classe ou unidade funcional é definida pelo que seus membros fazem - como agem ou funcionam - e não pela sua composição ou aparência. ... Uma classe ou categoria é chamada de "unidade" quando é tratada como um todo ... Skinner inventou o substantivo operante para ter um nome para uma categoria funcional de comportamento. ... Um operante é uma classe de atos que têm, todos, o mesmo efeito sobre o ambiente. (Baum, 1994/1997, pp 94-95).

Em contraposição, vejamos o atual posicionamento de Catania (1996), amplamente explicitado em um trabalho sobre as origens da estrutura do comportamento:

O operante, como uma classe de comportamento selecionada por suas consequências, é uma unidade fundamental do comportamento... Se a bicada de um pombo produz comida, por exemplo, bicar pode se estabelecer como um operante. Como operante, não pode ser confundido com classes de bicar que se originam de outras fontes (por exemplo, o bicar eliciado que é produzido pela automodelagem). A classe é definida em termos tanto de propriedades da resposta (por exemplo, a for- 
ça da bicada) quanto dos estímulos na presença dos quais a resposta ocorre (por exemplo, bicar o disco na presença de luz verde pode se estabelecer como um operante discriminado diferente de bicar o disco na presença de vermelho). O termo estímulo de um operante discriminado às vezes permanece implícito (por exemplo, bicar o disco depende de várias propriedades de estímulo do disco mesmo quando os estímulos permanecem constantes.

... é importante lembrar que a classe operante é definida por todos os três termos da contingência tríplice.

... classes operantes são criadas por contingências comuns, não por consequências comuns.

Classes operantes são definidas funcionalmente, não topograficamente. (Catania, 1996, pp 4-5).

Em resumo, a mesma resposta pode pertencer a classes operantes diferentes, consequências comuns não definem a mesma classe operante, topografias semelhantes podem ocorrer mas não são necessárias, pois a classe é definida funcionalmente. Duas instâncias pertencem à mesma classe operante quando todos os três termos das contingências são os mesmos, estímulo discriminativo, comportamento e conseqüência. As contingências são importantes tanto na distinção entre diferentes classes quanto na explicação do que mantem diferentes respostas unidas em uma mesma classe. Segundo Catania (1996), classes operantes podem ser produzidas de diferentes maneiras:

- Modelagem. A definição de resposta na contingência tríplice é alterada gradativamente, ocorrendo o reforço diferencial de aproximações sucessivas à resposta final - novo operante (Keller \& Schoenfeld, 1950). Exemplos: Eckerman, Hienz, Stein \& Kowlowitz, 1980; Platt, 1973; Stokes e Balsam, 1991.

- Desvanecimento (fading). Semelhantemente à modelagem da resposta podem ocorrer mudanças com aproximações sucessivas em relação à dimensões do estímulo discriminativo (Terrace, 1963). Uma nova classe operante se estabelece pela mudança do estímulo discriminativo da contingência tríplice.

- Estabelecimento de classes de ordem superior. Uma classe operante de nível superior inclui outras classes que podem funcionar como operantes por si. Exemplos:

1. Imitação de respostas que o imitador nunca viu antes (Poulson \& Kymissis, 1988).

2. Reforçamento de variabilidade no comportamento (Page \& Neuringer, 1985).

3. Reforçamento para novas respostas. Se o reforço for contingente à emissão de respostas que não foram reforçadas antes, respostas novas, não observadas antes no repertório, começam a aparecer (Pryor, Haag \& O'Reilly, 1969).

4. Classes definidas seqüencialmente, como no esquema de razão fixa (Ferster \& Skinner, 1957): em um esquema FR 10, somente a décima resposta de uma série é reforçada. $\mathrm{O}$ fator determinante do reforço é apenas a ordem em uma série, mas nove respostas não reforçadas são necessárias para que a décima o seja. Nos esquemas de reforçamento diferencial de diferentes tempos entre respostas (IRTs) o critério para o reforço é definido pelos limites mínimo e máximo para o tempo que separa duas respostas (Blough,1963, 1966; Shimp, 1967). Outro exemplo de classe definida seqüencialmente: Straub, Seidenberg, Bever e Terrace (1979).

5. No procedimento de escolha de acordo com o modelo (matching-to-sample), a escolha de uma cor dado que o modelo é daquela cor é um operante. Podemos ter um operante de ordem superior quando o sujeito escolhe acertadamente pares de cores novas (identity matching), nunca antes apresentadas (Catania, 1996). Da mesma forma, operantes de ordem superior estão envolvidos na equivalência de estímulos (Sidman, 1994).

6. No conceito de learning set temos classes operantes definidas por relações comuns a uma variedade de problemas de discriminação, não por estímulos específicos (Catania, 1996).

7. No caso do comportamento governado por regras, o seguir instruções é um operante de ordem superior (Skinner, 1969).

8. O comportamento verbal dá outros exemplos: mando, tato, autoclítico (Skinner, 1957).

Em resumo, depois de mais de meio século de progresso no aperfeiçoamento do conceito de unidade de análise, chegamos ao ponto em que a estrutura do comportamento é vista como inevitavelmente determinada por contingências. Catania (1996) assim coloca o estado da arte:

... reconhecer que as contingências que determinam o comportamento dependem, elas mesmas, da estrutura do ambiente é reconhecer o papel central e vital das contingências. Afinal, ao servir de mediador entre a estrutura do ambiente e a estrutura do comportamento, as contingências definem o próprio objeto de estudo da análise do comportamento. (Catania, 1996, p.10).

\section{Bibliografia}

Anger, D. (1956). The dependence of interresponse times upon the relative reinforcement of different interresponse times. Journal of Experimental Psychology, 44, 145-161.

Banaco, R.A. (1997). Sobre comportamento e cognição: aspectos teóricos, metodológicos e de formação em análise do comportamento e terapia cognitivista. Santo André: Arbytes.

Baum, W.M. (1994/1997). Compreender o behaviorismo. Trad. de M.T. Araújo Silva, M.A. Matos, G.Y. Tomanari e E.Z. Tourinho. Porto Alegre: Artes Médicas.

Blough, D.S. (1963). Interresponse time as a function of continuous variables: A new method and some data. Journal of the Experimental Analysis of Behavior, 6, 237-246.

Blough, D.S. (1966). The reinforcement of least-frequent interresponse times. Journal of the Experimental Analysis of Behavior, 9, 581-591.

Catania, A.C. (1973a). The psychologies of structure, function and development. American Psychologist, 28, 434-443. 
Catania, A.C. (1973b). The concept of the operant in the analysis of behavior. Behaviorism, 1, 103-116.

Catania, A.C. (1978). The psychology of learning: Some lessons from the Darwinian revolution. Annals of the New York Academy of Sciences, 309, 18-28.

Catania, A.C. (1988). Problems of selection and phylogeny, terms and methods of behaviorism. Em A.C. Catania e S. Harnand (Orgs.), The selection of behavior: The operant behaviorism of B. F. Skinner (pp. 474-483). New York: Cambridge University Press.

Catania, A.C. (1996). On the origins of behavior structure. Em T.R. Zentall e P.M. Smeets (org.), Stimulus class formation in humans and animals. New York: Elsevier. pp. 3-12.

Catania, A.C. (1998/1999). Aprendizagem: comportamento, linguagem e cognição. Trad. de D.G. Souza. Porto Alegre: Artes Médicas.

Eckerman, D.A., Hienz, R.D., Stern, S. \& Kowlowitz, V. (1980). Shaping the location of a pigeon's peck: effect of rate and siza of shapinf steps. Journal of the Experimental Analysis of Behavior, 33, 299-310.

Ferster, C.B. \& Skinner, B.F. (1957). Schedules of reinforcement. New York: Appleton-Century-Crofts.

Hayes, S.C., Gifford, E.V. \& Wilson, K.G. (1996). Stimulus classes and stimulus relations: arbitrarily applicable relational responding as an operant. Em T.R. Zentall \& P.M. Smeets (orgs.), Stimulus class formation in humans and animals. New York: Elsevier. Pp. 279-299.

Honig, W.K. (1966). Operant behavior: Areas of research and application. Englewood Cliffs, NJ: Prentice-Hall.

Honig, W.K. \& Staddon, J.E.R. (1977). Handbook of operant behavior. Englewood Cliffs, NJ: Prentice-Hall.

Keller, F.S. \& Schoenfeld, W.N. (1950). Principles of psychology. New York: Appleton-Century-Crofts.

Konorski, J. \& Miller, S. (1937). On two types of conditioned reflex. Journal of General Psychology, 16, 264-272.

Lettner, W.H. \& Rangé, B.P. (1988). Manual de psicoterapia comportamental. São Paulo: Manole.

Matos, M.A. (1999). Controle de estímulo condicional, formação de classes conceituais e comportamentos cognitivos. Revista Brasileira de Terapia Comportamental e Cognitiva, 1 (2), 159178.

Page, S. \& Neuringer, A. (1985). Variability is na operant. Journal of Experimental Psychology: Animal Behavior Processes, 11, 429-452.

Platt, J.R. (1973). Percentile reinforcement: paradigms for experimental analysis of response shaping. Em G. H. Bower (Org.), The psychology of learning and motivation. Vol. 7. New York: Academic Press.

Poulson, C.L. \& Kymissis, E. (1988). Generalized imitation in infants. Journal of Experimental Child Psychology, 46, 324-336.

Pryor, K.W., Haag, R. \& O'Reilly, J. (1969). The creative porpoise: training for novel behavior. Journal of the Experimental Analysis of Behavior, 12, 6533-661.

Schick, K. (1971). Operants. Journal of the Experimental Analysis of Behavior, 15, 413-423.

Sério, T.M.A.P. (1983). A noção de classe de respostas operante: sua formulação inicial. Dissertação de Mestrado não publicada, Instituto de Psicologia da Universidade de São Paulo.

Sério, T.M.A.P. (1990). Um caso na história do método científico; do reflexo ao operante. Tese de Doutorado não publicada, Instituto de Psicologia da Universidade de São Paulo.

Shimp, C.P. (1967). The reinforcement of short interresponse times. Journal of the Experimental Analysis of Behavior, 10, 425-434.
Sidman, M. (1960). Tactics of scientific research. New York: Basic Books.

Sidman, M. (1994). Equivalence relations and behavior: a research history. Boston, MA: Authors Cooperative.

Simonassi, L.E. (2001). Fazer, dizer e pensar: comportamentos operantes inter-relacionados. Anais do II Congresso Norte-Nordeste de Psicologia, Salvador, Bahia, maio de 2001.

Skinner, B.F. (1935a) The generic nature of the concepts of stimulus and response. Journal of General Psychology, 12, 40-65.

Skinner, B.F. (1935b). Two types of conditioned reflex and a pseudo type. Journal of General Psychology, 12, 66-77.

Skinner, B.F. (1937). Two types of conditioned reflex: A reply to Konorski and Miller. Journal of General Psychology, 16, 272279.

Skinner, B.F. (1938). The behavior of organisms. New York: D. Appleton-Century.

Skinner, B.F. (1953). Science and human behavior. New York: MacMillan. "Ciência e comportamento humano", tradução de J.C. Todorov e R. Azzi. Brasília: Editora Universidade de Brasília, 1967.

Skinner, B.F. (1957). Verbal behavior. New York: Appleton-Century-Crofts.

Skinner, B.F. (1966). Operant behavior. Em W.K. Honig (org.), Operant behavior: Areas of research and application. Englewood Cliffs, N.J.: Prentice-Hall. Pp. 12-32.

Skinner, B.F. (1969). Contingencies of reinforcement. A theoretical analysis. New York: Appleton-Century-Crofts.

Skinner, B.F. (1982). Sobre o behaviorismo. Trad. de Maria da Penha Villalobos. São Paulo: Cultrix/EDUSP. Trabalho original publicado em 1974.

Skinner, B.F. (1991). Questões recentes na análise do comportamento. Trad. de A.L. Néri. São Paulo: Papirus. Trabalho original publicado em 1989.

Stokes, P.D. \& Balsam, P.D. (1991). Effects of reinforcing preselected approximations of the rat's bar press. Journal of the Experimental Analysis of Behavior, 55, 213-231.

Straub, R.O., Seidenberg, M.S., Bever, T.G. \& Terrace, H.S. (1979). Serial learning in the pigeon. Journal of the Experimental Analysis of Behavior, 32, 137-148.

Teitelbaum, P. (1966). The use of operant methods in the assessment and control of motivational states. Em W. K. Honig (org.), Operant behavior: áreas of research and application. Englewood Cliffs, N.J.: Prentice-Hall.

Terrace, H.S. (1963). Errorless transfer of discrimination across two continua. Journal of the Experimental Analysis of Behavior, 6, 223-232.

Todorov, J.C. (1981). Behavior analysis and experimental pharmacology. Neuroscience and Biobehavioral Reviews, 5, 307-314.

Todorov, J.C. (1989). A psicologia como o estudo de interações. Psicologia: Teoria e Pesquisa, 3, 325-347.

Todorov, J.C. (1991). O conceito de contingência tríplice na psicologia experimental. Psicologia: Teoria e Pesquisa, 7, 5970 .

Verhave, T. (1966). The experimental analysis of behavior. New York: Appleton-Century-Crofts.

Recebido em 07.01.2002

Primeira decisão editorial em 10.08.2002

Versão final em 20.08.2002

Aceito em 02.09.2002 